\title{
CAPACIDAD DE CARGA Y PARÁMETROS VEGETACIONALES EN PRADERAS DEL HABITAT DE ALPACAS EN LA REGIÓN PUNO, CAMPAÑA 2018
}

LOAD CAPACITY AND VEGETATIONAL PARAMETERS IN ALPACAS HABITAT MEADOWS IN THE PUNO REGIÓN: 2018 CAMPAIGN

DOI: https://doi.org/10.47190/rcsaw.v2i3.7

Recibido: 03/01/22

Aceptado: 01/02/22
José Domingo Choquehuanca Soto Universidad Nacional de Juliaca jd.choquehuanca@unaj.edu.pe https://orcid.org/0000-0001-6269-3269

Edgar Pelinco Ruelas Universidad Nacional de Juliaca e.pelinco@unaj.edu.pe

RESUMEN https://orcid.org/0000-0003-3267-5611

Entre abril y agosto del 2018 en el predio Querotauca del distrito de Nuñoa, Melgar y región Puno, entre altitudes de 4,350 a 4,800 metros, se realizó un estudio con el objeto de evaluar la capacidad de carga y los parámetros vegetacionales en praderas del hábitat de alpacas. para la composición botánica y cobertura vegetal se empleó el método transecto al paso, la capacidad de carga animal actual se estimó en base a la producción de materia seca (MS) en relación al consumo de 1,300 gramos de MS/día de una alpaca macho reproductor de 65 kilogramos de peso vivo. Se analizo la cobertura y producción de MS, con correlaciones y regresiones lineales. Los resultados señalan que la capacidad de carga para épocas de lluvias y seca es 0.87 y $0.71 \mathrm{UAA} / \mathrm{ha}$, en forma respectiva, sin embargo, en las praderas en las épocas de lluvias y seca se encontraron en pastoreo 0.89 y $0.92 \mathrm{UAA} / \mathrm{ha}$, Las correlaciones y regresiones para la relación cobertura y producción forrajera en especies, resultan ser altamente significativas para Calamagrostis amoena, donde el incremento cobertural unitario aumenta en 0.72 gramos de MS y, para Festuca rigescens, incrementa 1.62 gramos. en conclusión, el predio esta sobrecapitalizado en 0.12 UAA/ha/año, y existe limitantes en producción forrajera en este tipo praderas nativas, teniéndose potencialidades que podrían revertir estas limitantes.

Palabras clave: Alpaca, cobertura vegetal, parámetros vegetacionales, praderas.

\begin{abstract}
Between April and August 2018 in the Querotauca property of the district of Nuñoa, Melgar and the Puno region, between altitudes of 4,350 to 4,800 meters, a study was carried out in order to evaluate the carrying capacity and the vegetative parameters in prairies of the habitat of alpacas. For the botanical composition and plant cover, the transect method was used, the current animal load capacity was estimated based on the production of dry matter (DM) in relation to the consumption of 1,300 grams of DM / day of a reproductive male alpaca of 65 kilograms of live weight. The coverage and production of MS was analyzed, with correlations and linear regressions. The results indicate that the carrying capacity for the rainy and dry seasons is 0.87 and 0.71 UAA / ha, respectively, however, in the grasslands in the rainy and dry seasons, 0.89 and 0.92 UAA / ha were found in grazing, The correlations and regressions for the coverage relationship and forage production in species, turn out to be highly significant for Calamagrostis amoena, where the unit coverage increase increases by 0.72 grams of DM and, for Festuca rigescens, increases 1.62 grams. In conclusion, the farm is overcapitalized by $0.12 \mathrm{UAA} / \mathrm{ha} /$ year, and there are limitations in forage production in this type of native grasslands, having potentialities that could reverse these limitations.
\end{abstract}

Keywords: Alpaca, vegetation cover, vegetational parameters, grasslands 


\section{INTRODUCCIÓN}

Una de las especies domesticas de camélidos sudamericanos de gran importancia para el Perú, es la alpaca (Vicugna pacos), que pasta en las praderas de la zona altoandina de la región Puno $y$, a nivel nacional es poseedor del mayor número de especies que se encuentra alrededor de 2 millones de cabezas, y representa el 59 \% de la población nacional. (CEPES, 2010).

Las praderas nativas a nivel nacional ocupan 19 millones de hectáreas, distribuidas en las zonas altas de la sierra y costa norte. De esta extensión, 13 millones de hectáreas, están en el sur del país (Astorga, 1984). Y, en la región de Puno, se disponen de 4 millones de hectáreas de praderas, aproximadamente, con una actividad de crianzas de ganado de altura (alpacas y llamas, principalmente). Ecosistemas, caracterizados por su inestabilidad climática (precipitación errática y presentación de temperaturas extremas) y un flujo de energía bastante limitado; factores que determinan que la ganadería sea más ventajosa que los cultivos. En consecuencia, la actividad humana en los ecosistemas de hábitat de camélidos sudamericanos, dependen mayormente de la productividad de forrajes producidos en las praderas. Donde la crianza de alpacas, básicamente se desarrolla a partir de los 3,900 a 4,600 metros de altitud, con diferentes sistemas de producción y tecnologías (tradicionales e intermedias), presentando estos sistemas de producción, índices de producción y productividad bajos, debido fundamentalmente al problema de la sobre carga animal y, el resultante sobrepastoreo de las praderas (Flórez et al, 1992).

En la actualidad, las praderas nativas de la zona altoandina de la región Puno, se encuentran sobrecapitalizadas; cuya consecuencia, se traduce en un estado de sobrepastoreo, al no adecuarse la especie animal y el número de animales por unidad de superficie en la pradera nativa. Las consecuencias, se tienen a la vista; disminución del potencial forrajero nativo, disminución de la cobertura, erosión de los suelos y por lo tanto baja producción y productividad de productos primarios de la actividad económica de la crianza de camélidos sudamericanos domésticos (fibra, pieles, carne, etc.). (Flórez, 2005).

El sobrepastoreo, es una práctica que evidencia el inadecuado manejo de la capacidad de carga de las praderas nativas; $y$, se origina en la idea errónea de prestar una mayor dedicación a los factores pág. 76 Waynarroque Revista de Ciencias Sociales Aplicadas zootécnicos del ganado domestico; $y$, no a los aspectos del medio ambiente. (Condori, 2005). Como es el componente de "Alimentación" en la crianza animal; base primordial en los sistemas de producción animal de alpacas; cuyo cimiento único es la producción de forrajes nativos en las praderas del pastizal nativo de la zona altoandina de la parte sur y centro del país. Esta información, es posible conjugarla sobre la base de un mapa del "Sitio de Pradera", que indiquen la "Capacidad de carga" y la "Condición de pradera". Que complementada, con la información obtenida de la presente investigación, permitirán trazar "Planes de manejo de praderas" adecuados; los que se traducirán en una mayor producción de productos primarios de origen animal por unidad de superficie; $y$, esencialmente, considerando la conservación del recurso natural (forrajes) en la pradera nativa. (Choquehuanca, 2004). La actividad económica de la crianza de alpacas en la zona altoandina de la región Puno, se viene desarrollando sin contar con "Planes de manejo". El mismo, que contempla conocer la capacidad de carga adecuada por tipo de pradera y superficie del predio. En base al comportamiento de la cubierta vegetal y la producción forrajera en la pradera nativa. (Zapana, 2020). En estas últimas décadas, la producción de forrajes en las praderas nativas ha venido decreciendo notablemente; el cual, se ve reflejado claramente, con la presencia de una vegetación con presencia de una retrogresión vegetal en las praderas nativas (Flórez et al, 1992).

La ejecución del estudio incluye la medición de los parámetros vegetaciones (cubertura vegetal y producción forrajera, principalmente); por cuanto el rendimiento productivo y reproductivo de la especie animal alpaca, ésta en función de la cantidad y calidad del forraje nativo disponible en la pradera nativa. (Zapana, 2020).

La investigación y la experiencia desarrollada en el sector han encontrado soluciones viables a la problemática de las praderas; tales como estimular el potencial productivo de las praderas, usar la especie, clase y el número adecuado de animales de acuerdo con la "Capacidad de carga" del predio, en consideración a las épocas de lluvias y seca. (Flores, Naupari, \& Tácuna, 2014).

Por lo general, se hace necesario que en sectores de los sistemas de producción de alpacas, principalmente; la identificación, el diseño, la adopción e implementación de tecnologías y 
actividades validadas en el componente de alimentación. Es base para el manejo y mejoramiento de praderas nativas, con el objeto primordial de incrementar la capacidad de carga por unidad de superficie en la región Puno. trabajos agrostológicos de investigación y validación de tecnologías de manejo de praderas nativas, demuestran que el problema general más serio y prevalente en la actualidad es el "inadecuado manejo de la capacidad de carga y los parámetros vegetacionales en praderas del hábitat de alpacas en la región Puno. (Mamani, 2012).

Institucionalmente, la investigación se justifica; ya que, el manejo del componente de alimentación de los camélidos sudamericanos domésticos, dependen del forraje producido en las praderas nativas. Donde, alpacas y llamas se alimentan directamente en la pradera; desconociéndose, el comportamiento estacional de la producción de forrajes, cobertura vegetal, composición florística, condición de la pradera, entre otros aspectos. Consecuentemente, se desconoce el comportamiento de la capacidad de carga por unidad de superficie en la pradera nativa. Razones por los cuales, los criadores de alpacas están a la espera de recomendaciones técnicas sobre los aspectos citados; la misma, que es producto de investigaciones reales y valederas obtenidas en el mismo campo. Constituyendo, la "Capacidad de carga", como uno de los aspectos principales en el manejo de praderas en la actualidad; por lo que se justifica la ejecución del presente trabajo de investigación. (Choquehuanca, 2004).

El presente trabajo, tiene por objeto evaluar la capacidad de carga y los parámetros vegetacionales en praderas del hábitat de alpacas. para la composición botánica y cobertura vegetal. El mismo, que conllevará a mejorar e incrementar la capacidad de carga por unidad de superficie a nivel de predio del criador de alpacas. Asu vez proponer y fomentar el manejo adecuado de la "Capacidad de carga" en las praderas nativas.

\section{MATERIALES Y MÉTODOS}

La investigación fue realizada en el predio rural de Querotauca, ubicada en el distrito de Nuñoa, provincia de Melgar y región de Puno. A una altitud, de 4,350 a 4,800 m.snm; y a una distancia de 45 km, de la capital del distrito (Nuñoa). Las coordenadas UTM del Centroide del predio es 8'420,185.5289 Norte y $338,177.0300$ Este.
La superficie total del predio rural es de 1,388.6091 hectáreas, donde el $100 \%$ de los terrenos de pastoreo están coberturados por pastos naturales y, en esta estructura natural, están inmersos diferentes tipos de praderas nativas utilizables; mayormente destinadas para la alimentación de alpacas, preferentemente. El predio rural, denominado "Querotauca", es propiedad de la familia Herrera Antezana y, está inscrito en las Oficinas de la SUNARP, Zona Registral XIII (sede Juliaca), con Ficha Registral No 2399.

\section{Análisis estadístico}

Las relaciones de los parámetros vegetacionales (cobertura vegetal y producción forrajera), se determinaron mediante el análisis de correlación y regresión lineal. Considerando la cobertura vegetal, como variable independiente $(\mathrm{X})$ y la producción forrajera, como variable dependiente $(Y)$. Coadyuvado con la función relacional de: $Y=f(X)$. Dando valores a $X(X 1, X 2, \ldots . . . X n)$, obtenemos valores correspondientes de $Y(Y 1, Y 2, \ldots . . . X n)$. Y, el desarrollo del "Modelo de regresión" (Steel y Torrie (1980), donde los valores esperados para la variable $Y$ de cualquier valor $X$, estará dado por la función lineal de: $Y=a+b X$. Donde: $Y$ (variable de respuesta o variable dependiente); $X$ (variable independiente o variable explicativa); y b (parámetros vegetacionales en estudio).

\section{Metodología aplicada}

Para obtener la producción forrajera, se hizo el reconocimiento ex-ante del predio rural, la identificación y evaluación de los tipos de pradera nativa en el predio, toma de muestras de la vegetación, en base a un tamaño de muestra (n) del $10 \%$ por tipo de pradera nativa identificado. En base al corte de la fitomasa aérea, con ayuda de un marco muestreador $(0.50 \mathrm{~m} 2)$, aplicando como criterio de uniformidad, el corte al ras del suelo (simulando el consumo al pastoreo de una alpaca). La fitomasa, cosechada, es depositada en una bolsa de plástico para su conservación y posterior secado en forma artificial. (Choquehuanca, 2004).

Paralelo a la cosecha de forraje y conocer la composición botánica y cobertura vegetal, se procedió a la lectura de la vegetación por tipo de pradera identificado en el predio y determinar la composición botánica y cobertura vegetal de las praderas nativas, mediante evaluaciones agrostológicas con el método de "Transección al paso" (Segura, 1963) Anexo 2).

Revista de Ciencias Sociales Aplicadas W Waynarroque pág. 77 
Para conocer la capacidad de carga (CC) y carga animal actual, que es definida como el máximo número de animales que puede soportar un área de pastoreo sin inducir o causar daños a la vegetación o recursos relacionados" (León e Izquierdo, 1993). se estimó en base a la producción de materia seca (MS) en relación al consumo de 1,300 gramos de MS/día, de una alpaca macho reproductor de 65 kilos de peso vivo (Leyva et al, 1988), en los periodos de abril y agosto del 2012; aplicando la formula siguiente:

$$
C C=\frac{D X f}{C X t}
$$

Donde:

CC : Capacidad de carga.

D : Producción primaria promedio de forraje seco (Kg, MS/ha).

$f$ : Factor de utilización del forraje, 50\% (0.50).

$C$ : Consumo de forraje por una unidad animal alpaca (Kg, MS/día).

$\mathrm{t}$ : Tiempo de utilización del potrero (en días).

La fórmula citada, para estimar la "Capacidad de carga" promedio, de camélidos sudamericanos por el método de consumo materia seca (MS) es directamente relacionada con los datos, que se detallan en la tabla 1.

Tabla 1

Dotos bose para estimar la capacidad de carga

\begin{tabular}{|c|c|c|c|}
\hline $\begin{array}{l}\text { LSPLCIL } \\
\text { ANIMAI }\end{array}$ & $\begin{array}{l}\text { PLSO VIVu } \\
\text { (Kilos, promedio) }\end{array}$ & $\begin{array}{l}\text { \% CONSUMM } \\
\text { (MS/PFSOVIVI) }\end{array}$ & $\begin{array}{l}\text { Consumo } \\
\text { (Ke, MS/dia) }\end{array}$ \\
\hline Alpaca & 65.00 & 2.00 & 1.3 \\
\hline Llama & 75.00 & 2.00 & 1.5 \\
\hline Vicuña & 38.5 & 2.60 & 1.00 \\
\hline
\end{tabular}

Fuente: Leyva et al (1988)

La carga animal actual, definida como el número de animales presentes en un área específica de pastoreo en un tiempo determinado. La misma, que se expresa en unidad animal o en unidades animal mes. La unidad animal considerada en el Perú es el equivalente a un ovino macho capón de 4 dientes de 35 kilogramos de peso vivo (Flórez y Bryant, 1989).

El estimado de la carga animal actual en pastoreo, se realizó mediante el uso de equivalencias a unidades animal ovino (UAO), mostrado en la tabla 2. Tomándose para propósitos de referencia, el animal tipo en la especie alpaca. "Un macho reproductor de 65 kilos de peso vivo". El mismo, que representa un animal tipo, para compararlo con las especies y clase de animal en pastoreo en el predio rural. Carga animal actual, que fue identificado y evaluado en el predio de Querotauca, durante los periodos de abril y agosto del 2018.
Tibla 2

Equivalencias de ganado domestico expresado en unidades animoi ovino (UAO)

\begin{tabular}{|c|c|c|c|}
\hline I specie animal & (lase animal & I dad (meses) & $\begin{array}{c}\text { I actor conversión } \\
\text { (UAO)" }\end{array}$ \\
\hline \multirow[t]{5}{*}{ Alpacas } & Padres & 24 a IIIas & 1.6 \\
\hline & Madres & 24 a mas & 1.5 \\
\hline & Tuis & 6 y 12 & 1.0 \\
\hline & Crias & Oab & 0.1 \\
\hline & lapones & 24 a mas & 1.6 \\
\hline \multirow[t]{5}{*}{ Llamas } & Padros & $24 \mathrm{a} \mathrm{mas}$ & 1.9 \\
\hline & Madies & 24 a Illas & 1.8 \\
\hline & Tuis & $6 \times 12$ & 1.2 \\
\hline & Críds & 0 a 6 & 0.9 \\
\hline & Caponies & 24 a Illas & 1.9 \\
\hline \multirow[t]{6}{*}{ Ovinus } & Cannelos & 18 d Illas & 1.0 \\
\hline & |3orregas & 18 a mas & 1.0 \\
\hline & |3orreguillas & 1) а 18 & 0.1 \\
\hline & (iarnerillos & 1) а 18 & 0.1 \\
\hline & Corderos & แล ๆ & 0.4 \\
\hline & Capones & 18 a mas & 1.2 \\
\hline \multirow[t]{4}{*}{ Vacurius } & Torus & 24 a Illas & 8.0 \\
\hline & Vacas & 24 a mas & S. 0 \\
\hline & Vaquillas & 12 a) & 4.0 \\
\hline & Inretes & $1 / \mathrm{A} / 1$ & 4.4 \\
\hline
\end{tabular}

(UAO)*: Unidad animal uvino (macho capón de 4 dienles de 35 kilus de peso vivo). I uente: I apia y I lórez (19k1)

\section{RESULTADOS}

Producción forrajera. La mayor producción de forraje nativo se obtuvo en la época de lluvias. Donde, el tipo de pradera "Césped de puna" mostró la mayor producción forrajera con 870 kilos de materia seca (MS) por hectárea (ha) para el periodo de abril, seguido por el tipo de pradera "Chilliguar húmedo" con 790 kilos de MS/ha (tabla 3).

Tabla 3

Disponibilidad forrajera por tipo de pradera en el postizal nativo del predio de querotouca (abril y ggosto, 2018)

\begin{tabular}{|c|c|c|c|}
\hline \multirow[t]{2}{*}{ Tipo praderu } & \multirow[t]{2}{*}{ Asociación vegetal } & \multicolumn{2}{|c|}{ Producción (MS, $\left.\mathrm{K}_{R} / \mathrm{Ha}\right)$} \\
\hline & & Abril 2018 & Aposto 2018 \\
\hline \multirow[t]{2}{*}{ Césped de puna } & Colamagrostis- & 870.00 & 720.00 \\
\hline & Scirpus & & \\
\hline \multirow[t]{2}{*}{ Chiliguar humedo } & Festuca & 190.00 & 630.00 \\
\hline & Caiamagrastis & & \\
\hline
\end{tabular}

Fucnte: Elaboración propia del lnvestigador.

Composición botánica y cobertura vegetal. La composición botánica que muestran los diferentes tipos de pradera nativa presentes en el predio de Querotauca, se detallan en la tabla 4 (abril, 2018) y tabla 5 (agosto, 2018). Donde se observa, que las especies forrajeras nativas más frecuentes, pertenecen a los géneros de: Calamagrostis, Festuca, Scirpus, Plantago, Hypochoeris, entre otros. Reportándose un total de 76 especies forrajeras nativas para la época de lluvias y, 55 especies para la época seca. Asimismo, se reporta una cobertura vegetal de $67.38 \%$ (época de lluvias) y $58.16 \%$ (época seca). Los cuales, en términos generales, revelan una condición de pradera de buena para la época de Iluvias y regular para la época seca (tabla 4 y 5 ). 
Tabla 4

Composición botánica y frecuencia de especies forrajeras en praderas del pastizal nativo del predio de Querotauca (abril, 2018)

\begin{tabular}{|c|c|c|}
\hline \multirow[t]{2}{*}{ Especies } & \multicolumn{2}{|c|}{ Tipos de pradera nativa } \\
\hline & Césped de puna* & Chilliguar húmedo* \\
\hline \multicolumn{3}{|l|}{ Deseables: } \\
\hline Agrostis breviculmis & 1.30 & 1.50 \\
\hline Polypogum elongatus & 0.67 & 0.50 \\
\hline Alchemilla erodiifolia & 0.50 & 0.25 \\
\hline Scirpus rigidus & 2.50 & - \\
\hline Valeriana $s p$ & 0.50 & 1.33 \\
\hline Plantago tubulosa & - & 3.50 \\
\hline Werneria nubigena & 0.15 & 0.50 \\
\hline Dissanthelium minimun & 0.50 & 0.10 \\
\hline Eleocharis albibracteata & 0.50 & 2.20 \\
\hline Carex spp & 1.00 & 3.00 \\
\hline Alchemilla pinnata & 0.67 & 2.00 \\
\hline Paspalum pigmaeum & 1.35 & 1.00 \\
\hline Poa spicigera & 0.67 & - \\
\hline Scirpus totorilla & - & 1.50 \\
\hline Gnaphalium capitatum & 0.50 & 2.00 \\
\hline $\begin{array}{l}\text { Luzula peruviana } \\
\text { L }\end{array}$ & 0.50 & 0.67 \\
\hline Werneria spp & 1.50 & 0.50 \\
\hline Bromus lanatus & 0.50 & 1.33 \\
\hline Gnaphalium sp & 0.50 & - \\
\hline Hypochoeris $s p$ & - & 1.67 \\
\hline Hypochoeris stenocephala & 0.67 & - \\
\hline Hypochoeris radicata & 0.25 & - \\
\hline Trifolium peruvianun & 0.67 & 0.50 \\
\hline Eleocharis retroflexa & - & 1.50 \\
\hline Geranium sessiliflorum & 0.50 & 2.50 \\
\hline Geranium $s p$ & 0.25 & 0.50 \\
\hline Dissanthelium $s p$ & 0.50 & \\
\hline Bromus sp & 0.25 & 1.00 \\
\hline Distichia muscoides & - & 2.50 \\
\hline Stylitis andicola & - & 1.50 \\
\hline Mimulus glabratus & $\ldots$ & 0.50 \\
\hline
\end{tabular}

Poco deseables:

Calamagrostis amoena

Calamagrostis rigescens

Stipa spp

Stipa inconspicua

Stipa hans-meyer

Calamagrostis rigida

Calamagrostis $s p$

Calamagrostis

Stipa obtusa

Oreomyrrhis sp

Cyperus sp

Cymbergia peruviana

Liavum ovatum

Lepechenia meyeni

Stipa brachyphyll

Juncus balticus

Senecio $s p$
Oreomirrhys andicola

Calamagrostis antoniana

Ranunculus sp

Azorella bilovata

Lucilia microphylla

Lucilia aretiodes

Nothoscordium flabellato

Lucilia sp

Castilleja sp

Perezia sp

Sisyrrinchium $s p$

Arenaria $s p$

Paronichia andina

Cerastium sp

Perezia coerulescen

Festuca peruviana

Calamagrostis mínima

3.50

3.50
-
1.00
1.67

1.00
1.67

1.33

1.50

0.33

1.00

1.33

1.33
0.50

0.33

1.00
0.67

$\begin{array}{ll}0.50 & - \\ 0.50 & 1.00\end{array}$

$\begin{array}{ll}0.50 & 1.50 \\ - & 1.50\end{array}$

1.50

1.50

1.00

$1.50-0.50$

$\begin{array}{ll}.50 & 0.50 \\ 1.50 & 0.50\end{array}$

$\begin{array}{ll}.50 & 0.50\end{array}$

0.50

$\begin{array}{ll}.00 & 1.50 \\ .00 & 0.20\end{array}$

$\begin{array}{ll}.50 & 0.10 \\ .00 & 1.50\end{array}$

.50

1.50

1.00

0.50

\begin{tabular}{lll}
0.50 & 0.50 \\
\hline
\end{tabular}

\section{Pygnophyllium spp}

Aciachne pulvinata

Astragalus microphylla

Lugonia sp

Plantago linearis

Astragalus dielsii

Denothera multicaulis

Cotula sp

Plantago rigescens

Azorella pulvinata

Azorella diapensoides

Cobertura vegetal

$\begin{array}{ll}3.50 & 0.33 \\ 3.50 & 1.50\end{array}$

$3.50 \quad 1.50$

0.10

0.67
2.50

2.50
1.50

$50-50$

1.50

1.75
0.25

$\begin{array}{ll}0.75 & 1.50 \\ 6.50\end{array}$

* Calamagrostis - Scirpus.

** Festuca-Calamagrostis.

Fuente: Elaboración propia del Investigador.
Tabla 5

Composición botánica y frecuencia de especies forrajeras en praderas del pastizal nativo del predio de Querotauca (agosto, 2018)

\begin{tabular}{|c|c|c|}
\hline \multirow[t]{2}{*}{ Especies } & \multicolumn{2}{|c|}{ Tipos de pradera nativa } \\
\hline & Césped de puna* & Chilliguar húmedo* \\
\hline \multicolumn{3}{|l|}{ Deseables: } \\
\hline Agrostis breviculmis & 1.00 & 1.50 \\
\hline Alchemilla erodiffolia & 0.10 & 0.25 \\
\hline Scirpus rigidus & 4.00 & - \\
\hline Plantago tubulosa & - & 4.50 \\
\hline Dissanthelium minimun & 0.50 & 0.10 \\
\hline Eleocharis albibracteata & - & 2.00 \\
\hline Carex spp & - & 1.50 \\
\hline Alchemilla pinnata & - & 1.50 \\
\hline Poa spicigera & 0.67 & 0.50 \\
\hline Scirpus totorilla & - & 1.50 \\
\hline Gnaphalium capitatum & 0.10 & 0.10 \\
\hline Luzula peruviana & 1.10 & 0.50 \\
\hline Gnaphalium sp & 0.50 & - \\
\hline Hypochoeris sp & - & 2.50 \\
\hline Trifolium peruvianun & - & 0.50 \\
\hline Eleocharis retroflexa & - & 1.50 \\
\hline Geranium sessiliflorum & 0.50 & 0.10 \\
\hline Geranium sp & 0.10 & 0.50 \\
\hline Bromus sp & - & 1.00 \\
\hline Distichia muscoides & - & 2.50 \\
\hline Stylitis andicola & - & 1.50 \\
\hline Mimulus glabratus & - & 0.50 \\
\hline
\end{tabular}

Mimulus glabratus

Poco deseables:

Calamagrostis amoena

Calamagrostis rigescens

Stipa spp

Stipa inconspicua

Stipa hans-meyeri
Festuca rigescens

Calamagrostis rigida

Calamagrostis sp

Stipa obtusa

Oreomyrrhis sp

Cyperus $s p$

Liavum ovatum

Stipa brachyphylla

Juncus balticus

Oreomirrhys andicola

Calamagrostis antoniana

Ranunculus sp

Azorella bilovata

Sisyrrinchium $S p$

Arenaria sp

Festuca peruviana

Calamagrostis minima

Indeseables:

Pygnophyllium spp

Aciachne pulvinata
Astragalus microphylla

Lugonia sp

Plantago linearis

Astragalus dielsii

Denothera multicaulis

Plantago rigest

Azorella pulvinata

Azorella diapensoides

Cobertura vegetal

* Calamagrostis - Scirpus.

** Festuca-Calamagrostis.

Fuente: Elaboración propia del Investigador.

\section{RESULTADOS}

Producción forrajera. La mayor producción de forraje nativo se obtuvo en la época de lluvias. Donde, el tipo de pradera "Césped de puna" mostró la mayor producción forrajera con 870 kilos de materia seca (MS) por hectárea (ha) para el periodo de abril, seguido por el tipo de pradera "Chilliguar húmedo" con 790 kilos de MS/ha (tabla 3).

Tabla 6

Coeficiente de correlación (r) y regresión (b) para cobertura vegetal y producción forrajera de especies predominantes por tipo de pradera nativa (abril, 2018)

Tipo pradera Asociación vegetal Cobertura (\%) y producción de MS (gr/050

\begin{tabular}{|c|c|c|c|c|}
\hline & & \multicolumn{3}{|l|}{$\mathrm{m} 2)$} \\
\hline & & $r$ & $r^{2}$ & $Y=a+b X$ \\
\hline Césped de puna & Calamagrostis -Scirpus & $0.6837^{* *}$ & 0.4674 & $Y=-1.98+0.72 X$ \\
\hline \multicolumn{5}{|l|}{ Chilliguar húmedo } \\
\hline & Festuca-Calamagrostis & $05627^{* *}$ & 0.3166 & $Y=2.58+1.62 X$ \\
\hline
\end{tabular}

*= Significativo para P: 0.05

** = Significativo para P: 0.01

Fuente: Elaboración propia del Investigador.

Revista de Ciencias Sociales Aplicadas 
La capacidad de carga de las praderas es de $0.87 \mathrm{y}$ 0.71 UAA/ha, para las épocas de lluvias y seca, en forma respectiva (tabla 7 y 8). Sin embargo, en las praderas en las épocas de lluvias y seca se encontraron en pastoreo 0.89 y 0.92 UAA/ha, respectivamente (tabla 9 y 10). Lo que nos revela, que las praderas del predio están sobre-capitalizadas en $0.12 \mathrm{UAA} / \mathrm{ha} / a n ̃ o$. Lo que equivale a decir, que existen 156 alpacas que están de más, pastoreando las praderas nativas del predio. Resultados que nos revelan que en las dos épocas (lluvias y seca) de la campaña del año 2018, existen diferencias significativas en la capacidad de carga $y$, diferencias entre los tipos de pradera de césped de puna y Chilliguar húmedo; donde el tipo de pradera "Césped de puna", mostró la mayor capacidad de carga por unidad de superficie de pastoreo con 0.92 y 0.76 UAA/ha para las épocas de lluvias y seca, en forma respectiva (tabla 7 y 8 ).

Tabla 7

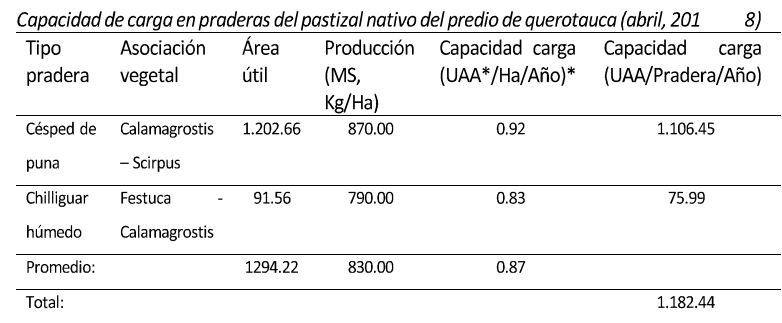

\section{Tabla 8}

Capacidad de carga en praderas del pastizal nativo del predio de querotauca lagosto, 2018)

\begin{tabular}{llcccc}
$\begin{array}{l}\text { Tipo } \\
\text { pradera }\end{array}$ & $\begin{array}{l}\text { Asociación } \\
\text { vegetal }\end{array}$ & $\begin{array}{l}\text { Área } \\
\text { útil }\end{array}$ & $\begin{array}{l}\text { Producción } \\
\text { (MS, } \\
\mathrm{Kg} / \mathrm{Ha})\end{array}$ & $\begin{array}{l}\text { Capacidad carga } \\
\text { (UAA*/Ha/Año)* }\end{array}$ & $\begin{array}{l}\text { Capacidad carga } \\
\text { (UAA/Pradera/Año) }\end{array}$ \\
\hline $\begin{array}{l}\text { Césped de } \\
\text { puna }\end{array}$ & $\begin{array}{l}\text { Calamagrostis } \\
\text { - Scirpus }\end{array}$ & 1.202 .66 & 720.00 & 0.76 & 914.02 \\
\hline $\begin{array}{l}\text { Chilliguar } \\
\text { húmedo }\end{array}$ & $\begin{array}{l}\text { Festuca } \\
\text { Calamagrostis }\end{array}$ & 91.56 & 630.00 & 0.66 & 60.43 \\
\hline Promedio: & & 1294.22 & 675.00 & 0.71 & \\
\hline Total: & & & & & \\
\hline
\end{tabular}

UAA*: Unidad Animal Alpaca (macho reproductor de 65 kilos de peso vivo).

Fuente: Elaboración propia del Investigador

Tabla 9

Carga animal actual en pastoreo en praderas del pastizal nativo del predio de querotauca (abril, 2018)

\begin{tabular}{lcccc}
\hline Especie animal & Clase animal & Cabezas ganados & $\begin{array}{l}\text { Factor de } \\
\text { conversión (uao)* }\end{array}$ & $\begin{array}{l}\text { Total conversión } \\
\text { (uaa)** }\end{array}$ \\
\hline Alpaca & Padres & 97 & 1.6 & 97 \\
& Madres & 576 & 1.5 & 540 \\
& $\begin{array}{c}\text { Tuis machos } \\
\text { Tuis hembras }\end{array}$ & 167 & 1.0 & 105 \\
& Crías & 187 & 1.0 & 117 \\
& 208 & 0.7 & 91 \\
\hline Vacuno & Toros & 1 & & 5 \\
& Vacas & 20 & 5.0 & 63 \\
& Vaquillas & 5 & 5.0 & 13 \\
& Toretes & 6 & 4.0 & 17 \\
& Ternero(a) & 9 & 2.4 & 14 \\
\hline Ovino & Carneros & 2 & 1.0 & 2 \\
& Borregas & 97 & 1.0 & 61 \\
& Borreguillas & 34 & 0.7 & 15 \\
& Carnerillos & 26 & 0.7 & 12 \\
\hline Total & & 1435 & & 1152 \\
\hline
\end{tabular}

(UAO)* : Unidad Animal Ovino (macho capón de 4 dientes de 35 kilos de peso vivo). (UAA)**: Unidad Animal Alpaca (macho reproductor de 65 kilos de peso vivo). Fuente: Elaboración propia del Investigador.
Tabla 10

Carga animal actual en pastoreo en praderas del pastizal nativo del predio de querotauca (agosto, 2018)

\begin{tabular}{lcccc}
\hline Especie animal & Clase animal & Cabezas ganados & $\begin{array}{l}\text { Factor de } \\
\text { conversión (uao)* }\end{array}$ & $\begin{array}{l}\text { Total conversión } \\
\text { (uaa)** }\end{array}$ \\
\hline Alpaca & Padres & 97 & 1.6 & 97 \\
& Madres & 576 & 1.5 & 540 \\
& Tuis machos & 271 & 1.0 & 170 \\
& Tuis hembras & 291 & 1.0 & 182 \\
\hline Vacuno & Toros & 1 & 8.0 & 5 \\
& Vacas & 20 & 5.0 & 63 \\
& Vaquillas & 5 & 4.0 & 13 \\
& Toretes & 6 & 4.4 & 17 \\
& Ternero(a) & 9 & 2.4 & 14 \\
\hline Ovino & Carneros & 2 & 1.0 & 2 \\
& Borregas & 97 & 1.0 & 61 \\
& Borreguillas & 34 & 0.7 & 15 \\
& Carnerillos & 26 & 0.7 & 9 \\
\hline Total & & 1429 & & 1188 \\
\hline
\end{tabular}

Fuente: Elaboración propia del Investigador.

Tabla 11

Interacción de la capacidad de carga y carga animal actual en praderas del pastizal nativo del predio de querotauca (abril y agosto, 2018)

\begin{tabular}{cccc}
\hline Estación del año & $\begin{array}{c}\text { Capacidad de carga } \\
\text { (UAA } / \mathrm{Ha} / \mathrm{Anño}^{\circ}\end{array}$ & $\begin{array}{c}\text { Carga animal actual } \\
\left(\mathrm{UAA}^{*} / \mathrm{Ha} / \mathrm{Anñ}^{\circ}\right)\end{array}$ & $\begin{array}{c}\text { Interacción } \\
\left(\mathrm{UAA}^{*} / \mathrm{Ha} / \mathrm{Anño}\right)\end{array}$ \\
\hline Luvias (abril) & 0.87 & 0.89 & Mas de 0.002 \\
\hline Seca (agosto) & 0.71 & 0.92 & Mas de 0.21 \\
\hline Promedio: & 0.79 & 0.91 & Mas de 0.12
\end{tabular}

(UAA*): Unidad Animal Alpaca (macho reproductor de 65 kilos de peso vivo). Fuente: Elaboración propia del Investigador

\section{DISCUSIÓN}

Al respecto (FAO, 1996), reporta que la producción forrajera anual de las praderas del área de estudio pudo ser estimada mediante la implementación de un modelo de simulación que utiliza variables climáticas medias y una producción potencial, en un entorno SIG raster; si bien no fue validada, la producción estimada retrata satisfactoriamente las tendencias de la zona de estudio, crecimiento concentrado en los periodos de lluvias y, mayor producción forrajera en la pradera tipo "Bofedal" (FAO, 1996). Sin embargo, este hecho no puede ser sobre enfatizado, porque la causa más importante de la pobre productividad animal en el Perú es la inadecuada nutrición por la baja cantidad y/o pobre calidad de los forrajes producido en las praderas nativas (San Martin, 1991).

Huisa (1996) en una evaluación agrostológica de una pradera tipo "Bofedal" del Centro de Investigación La Raya de la UNSAAC Cusco, identifico un total de 24 especies vegetales para la época de lluvias, con una cobertura vegetal de $96 \%$, los mismos que están representados en mayor porcentaje por especies forrajeras, como: Distichia muscoides (19\%), Plantago tubulosa (16\%), Lucilia aretiodes $(11 \%)$, Alchemilla diplophylla (10\%), Lilaeopsis andina (9\%), entre otras especies (31\%) y, en cambio para la época seca, reporto una cobertura vegetal del orden de $70 \%$, donde las especies que representan el mayor porcentaje de cobertura son: Distichia muscoides (27\%), Plantago tubulosa (17\%), Lucilia aretiodes (10\%), Lilaeopsis andina (8\%) y Alchemilla diplophylla (8\%). En cambio, Flórez (1972) como resultado del 
estudio más completo realizado en el Perú sobre cargas y sistemas de pastoreo en pastizales del altiplano de Puno, obtuvo cambios negativos en la composición botánica de la vegetación nativa, así como menor producción por cabeza con carga altas (3 ovinos/ha), al compararse con cargas ligeras (1.5 ovinos/ha).

El coeficiente de determinación de $r 2=0.4674$, para Calamagrostis amoena (asociación CalamagrostisScirpus), implica que sólo el $46.74 \%$ de la producción de MS, obedece al incremento de la cobertura de esta especie; y el resto se debería a factores no determinarlos en el presente estudio. Para el caso de la especie Festuca rigescens (asociación FestucaCalamagrostis), este coeficiente es menor (31.66\%); donde la fracción restante, se debe a otros factores ajenos a la variable cobertura. Aunque, estas relaciones no gozan de una aceptación generalizada, debido a que normalmente las plantas de crecimiento bajo cubren a menudo áreas mayores que las especies de crecimiento alto (Stoddart et al, 1975). Estos resultados estarían influenciados por el estado sucesional, incidiendo una progresión luego de un sobrepastoreo de la pradera. De manera, que la ganancia en cobertura vegetal, significaría un mayor macollamiento y un incremento de forraje nativo (Sosebee, 1982).

Marez (1972) en un trabajo similar en la Sierra Central del Perú, obtuvo una capacidad de carga óptima de 2 ovinos/ha, bajo pastoreo continuo; sin embargo, estos resultados son solo de un año productivo y, por lo tanto, no confiables para efectos de análisis concienzudo. Lo anterior fue comprobado por Sánchez (1975), quien después de tres años de estudio concluyo que la capacidad de carga optima era 3 ovinos/ha, carga que presentaba mejores ganancias, así como la persistencia de una buena condición de las praderas nativas.

Sobre tema Flórez y Malpartida (1992), reportan para Pampa Galeras con 6,500 hectáreas, relacionando con la población de vicuñas, la proporción de la capacidad de carga es de 1.15 unidades animal vicuña (UAV) por hectárea (ha); pero, considerando las otras especies en pastoreo en la superficie de las 6,500 hectáreas (alpacas, ovinos, llamas y vacunos) y convirtiendo estos a unidades animal vicuñas, se obtiene un equivalente a 8,589 UAV; la misma que relacionando con el área total $(6,500$ hectáreas), presenta una proporción actual de 1.32 UAV/ha. Lo que demuestra que el estudio realizado en Pampa Galeras, presenta un serio problema de sobre-capitalización (Flórez y Malpartida, 1992). Sin embargo Choquehuanca (2004), evaluando praderas nativas del Comité Multicomunal de Manejo de Vicuñas de Cala Cala (provincia de San Antonio de Putina, región de Puno), determino que existen diferencias significativas en la "Capacidad de carga" entre los potreros de Quellocunca, Tuturpunco, Ccamañani, Tisñamocco, Ticllacocha y Payanicunca en las épocas de Iluvias y estiaje; donde estimó una capacidad de carga de 3,227.38 unidades animal vicuña (UAV)/cerco/año en la época de lluvias y de 2,344.46 UAV/cerco/año en la época de estiaje. No obstante, en las praderas se encontraron en pastoreo 1,307 UAV (época de lluvias) y 1,203 UAV (época de estiaje); interacción que demuestra, que las praderas nativas del Comité de Cala Cala, se encuentran descapitalizadas en el orden de - 1,920.38 UAV y de - 1,141.46 UAV para las épocas de lluvias y estiaje, en forma respectiva (Choquehuanca, 2004). Resultados que demuestran que la capacidad de carga, está supeditado a la producción forrajera de la época de lluvias, ya que durante los periodos de la época de estiaje, la disponibilidad de forrajes se reduce considerablemente, por lo que el manejo del pastoreo debe estar dirigido a la restricción del tiempo de pastoreo en las praderas en los periodos críticos de forrajes, sobre todo el máximo aprovechamiento del pasto disponible en las áreas que se rotan en la época de lluvias por su relación con la disponibilidad y calidad nutricional en los ciclos posteriores de pastoreo, lo cual se facilita con el empleo del pastoreo fraccionado (Álvarez, 1965). Al respecto Gamarra et al (1985) establece que los factores principales que afectan la productividad de los pastos nativos son el sistema de pastoreo, la clase de ganado y la carga animal; afirmando que el factor carga animal es el más importante de los tres factores. Las ganancias de peso en los animales permanecerán constantes hasta un punto de equilibrio, determinado por la carga animal en el cual el total de forraje utilizable equivale al forraje consumido, asumiendo que todos los animales están pastoreando a su máximo; más allá de este punto la ganancia por animal esta inversamente relacionada con la carga animal (Gamarra et al, 1985). Al respecto (Pezo et al, 1992) manifiestan que la capacidad de carga de un predio, varia cada año, dependiendo principalmente de la precipitación pluvial y el grado de utilización por el ganado en pastoreo del año anterior; por lo tanto, se recomienda también considerar la capacidad de carga de años normales y tener la flexibilidad para reducir la carga animal actual en las praderas del predio. Revista de Ciencias Sociales Aplicadas W aynarroque| pág. 81 
La carga animal actual en pastoreo en las praderas del predio "Querotaauca" en las épocas de lluvias y seca, se reportan en las (tablas 9 y 10). La carga animal, que mayormente son de la especie y clase animal de alpacas (padres, madres, tuis machos, tuis hembras y crías); vacunos (toros, vacas, vaquillas, toretes, terneras y becerros); y ovinos (carneros, borregas, borreguillas y carnerillos). En trabajos similares (Pezo et al, 1992) en manejo de pastoreo y carga animal con novillos por unidad de hectárea, encontró que la carga animal afecta negativamente la disponibilidad forrajera, indicando que existe un rango de carga animal "Optimo", obteniéndose una aceptable ganancia de peso por animal por hectárea, estabilidad ecológica y rentabilidad económica. Y, la FAO (1996), indica que la carga animal adecuada permite cuantificar el grado en que una pradera está siendo sobre-utilizada; información que usada conjuntamente con otros elementos (adecuación a distintas categorías animales y época de crecimiento) es base para tomar decisiones de manejo del ganado en el predio.

Sobre la interacción de la capacidad de carga optima y carga animal actual en el predio de "Querotauca", se tuvo que la capacidad de carga fue de 0.87 y 0.71 UAA/ha, para las épocas de lluvias y seca, respectivamente (tabla 11).

Sin embargo, en las praderas pastorean 0.89 y 0.92 $\mathrm{UAA} / \mathrm{ha}$, en las épocas de lluvias y seca, en forma respectiva. Resultados, que revelan que las praderas se encuentran sobre-capitalizadas en 0.02 y 0.21 UAA/ha, en las épocas de lluvias y seca, respectivamente (tabla 11). Praderas que, en promedio anual, se encuentran sobre-capitalizadas en 0.12 UAA/ha/año; lo que equivale a decir, que existe 156 unidades animal alpaca (UAA), de más, que están pastoreando las praderas del predio de Querotauca.

\section{CONCLUSIONES}

La capacidad de carga de las praderas es de 0.87 y 0.71 UAA/ha, para las épocas de lluvias y seca, en forma respectiva. Sin embargo, en las praderas en las épocas de lluvias y seca se encontraron en pastoreo 0.89 y 0.92 UAA/ha, respectivamente. Revelando, que las praderas del predio están sobre-capitalizadas en 0.12 UAA/ha/año. Lo que equivale a decir, que existen 156 alpacas que están de más, pastoreando las praderas nativas del predio.

Las correlaciones y regresiones para la cobertura vegetal y la producción forrajera de las especies predominantes "Tipo" de las praderas nativas, resultan ser altamente significativas; mostrando para Calamagrostis amoena de la asociación Calamagrostis - Scirpus, el incremento de la cobertura de una unidad porcentual, se traduce en un incremento de 0.72 gramos de MS; en cambio para Festuca rigescens de la asociación Festuca Calamagrostis, el incremento es de 1.62 gramos de MS por el incremento de una unidad de cobertura; asimismo, las 2 especies "Tipo", muestran coeficientes de determinación (r2) menores a 0.50 .

\section{REFERENCIAS BIBLIOGRÁFICA}

Astorga, J. (1984). Algunos problemas en el manejo de praderas para la producción ganadera en el altiplano peruano. Copia mimeografiada. UNTA - Puno, Perú.

CEPES, (2010). Diagnóstico situacional de los camélidos en la región de Puno. [Internet], [4 febrero 2021]. Disponible en: h t t p://w w w. cepes.org.pe/ cendoc/Propuesta_candidato_region_Puno.p df

Condori, L. (2005). Evaluación agrostologica y alternativas de mejoramiento y manejo de pastos y forrajes del fundo Los Sauces S.A.C. Muñani. Azángaro. Tesis Ing. Agr. Puno. Perú.

Choquehuanca, J.D. (2004). Caracterización agrostológica de la capacidad de carga en praderas nativas de Cala Cala - Puno. Tesis de Maestría. Escuela de Post Grado. Universidad Nacional del Altiplano. Puno, Perú.

FAO. (1996). El Manejo de las praderas relacionado con la ecología. Principios de manejo de praderas naturales. Oficina regional de la FAO para América Latina y el Caribe. Capítulo VI. Santiago, Chile.

Farfán, R.; y Duránt, A. (1998). Manejo y técnicas de evaluación de pastizales altoandinos. Estación Experimental Maranganí. Instituto Veterinario de Investigaciones Tropicales y de Altura (IVITA) - La Raya. Universidad Nacional Mayor de San Marcos. Cusco, Perú.

Florez, A. (2005). Manual de pastos y forrajes altoandinos ITDG al OIKOS. Lima. Perú. 53p 
Flórez, A. (1972). Sistemas de pastoreo en pastizales nativos en el altiplano peruano. Boletín $N^{\circ} 11$. Programa de Forrajes - UNALM. Lima, Perú.

Flórez, A.; y Bryant, F. (1989). Manual de pastos y forrajes. Dirección Regional de Investigación Pecuaria. Instituto Nacional de Investigación Agropecuaria y Agroindustrial (INIAA). Lima, Perú.

Flórez, A.; Malpartida, E.; y San Martin, F. (1992). Manual de forrajes para zonas áridas y semiáridas andinas. Red de rumiantes menores. Convenio Universidad de California, Davis - Instituto Nacional de Investigación Agropecuaria y Agroindustrial (INIAA). Lima, Perú.

Flores, E.R., Ñaupari, J.A., y Tácuna, R.E. (2014). La economía del cambio climático en el Perú: ganadería altoandina. En: La economía del cambio climático en el Perú. Banco Interamericano de Desarrollo, Comisión Económica para América Latina y el Caribe. R e c u p e r a d o d e http://repositorio.cepal.org/bitstream/handl e/11362/37419/S1420992 es.pdf;jsessionid =6A859F66F15CDA337155DD749BC2B74B? sequence $=1$

Gamarra, J.; Flórez, A.; Bryant, F.; Schulundt, A.; y Malpartida, E. (1985). Producción ovina bajo tres sistemas de pastoreo en la sierra central del Perú. En: L.C. Fierro y R. Farfán, eds. Investigación sobre pastos y forrajes. Texas Tech University en el Perú. Articulo técnico T9-408.

Huisa, T. (1996). Pastizales y nutrición al pastoreo. Centro Experimental y Producción - La Raya. Programa de Ganadería Andina. Edit. Universidad Nacional San Antonio Abad del Cusco (UNSAAC). Cusco, Perú.

Mares, V. (1972). Determinación de la capacidad de soporte de algunos pastizales nativos altoandinos mediante el sistema de cargas fijas. Tesis Universidad Nacional Agraria - La Molina. Lima, Perú.
Mamani, L. 2012. estudio económico de la producción de alpacas en las comunidades de puna seca. Universidad Nacional del Altiplano. Tesis de post grado maestría en $\mathrm{g}$ a $\mathrm{n}$ a d e ría a $\mathrm{nd} \mathrm{i} \mathrm{n}$ a. http://repositorio.unap.edu.pe/handle/UNA $\underline{P} / 352$

Leyva, V.; Núñez, A.; Jiménez, J.; Choquehuanca, J.D.; y Franco, E. (1988). Sistemas de producción de camélidos sudamericanos. Informe de la VIII Reunión general de la Red de Investigación en Sistemas de Producción Animal en Latinoamérica (RISPAL). Ed. por M.E. Ruíz y A. Vargas. IICA/RISPAL. San José, Costa Rica.

León, C.; y Izquierdo, F. (1993). Producción y utilización de los pastizales de la zona altoandina. Compendio. Red de pastizales andinos. Proyecto con apoyo del Centro Internacional de Investigación para el Desarrollo, CIID - Canadá. 1ra. Edición. Quito, Ecuador.

Pezo, D.; Romero, F.; y Ibrahim, M. (1992). Producción, manejo y utilización de los pastos tropicales para la producción de carne. Avances en la producción de carne en el trópico americano. Oficina Regional de la FAO para América Latina y el Caribe. Santiago, Chile.

Steel, RGD y Torrie, JH (1980). Principios y procedimientos de estadísticas. Un enfoque biométrico. $2^{a}$ edición. McGraw-Hill, Nueva York, EE. UU., Págs. 20-90.

Segura, M. (1963). Evaluación de la productividad de los campos forrajeros de puna. Informe especial $N^{\circ}$ 3. Editorial OTIA - SIPA. Lima, Perú.

Stoddart et al. (1975) Range management. Third edition. New York, United States.

Sosebee, D. (1982). Manejo y mejoramiento de pastizales nativos. Texas Tech University. Programa Colaborativo de Apoyo a la Investigación en Rumiantes Menores. Curso corto. Lima, Perú.

Steel, R.G.; y Torrie, J.H. (1980). Principles and procedures of statistics. 2nd, ed. McGraw Hill book, Co. New York, United States. 
San Martin, F. (1991). Nutrición y alimentación de alpacas y llamas. Producción de Rumiantes Menores - Alpacas. Edic. Novoa, C. y Flórez, A. Convenio Universidad de California Davis INIAA. Lima, Perú.

Tapia, M.; y Flórez, A. (1984). Pastoreo y pastizales de los andes del sur del Perú. Instituto Nacional de Investigación y Promoción Agropecuaria (INIPA). Edit. Arteta. Lima, Perú.

Zapana Landaeta, J. C. (2020). Evaluación de pastizales naturales y determinación de la carga animal actual en la comunidad chila, Puno. Perú. Revista De Investigaciones De La Escuela De Posgrado De La UNA PUNO, 8(4), 1286 - 1296. Recuperado a partir de http://revistas.unap.edu.pe/epg/index.php/in vestigaciones/article/view/1280 